\title{
Detecção de Doenças Sexualmente Transmissiveis em Clínica de Planejamento Familiar da Rede Pública no Brasil
}

\author{
STD Screening in a Public Family Planning Clinic in Brazil \\ José Santiago de Codes ${ }^{1}$, Deborah Ann Cohen ${ }^{2}$, Neli Almeida de Melo ${ }^{1}$, \\ Alessandra Barbosa Santos ${ }^{1}$, João Jorge Góes de Codes ${ }^{1}$, \\ Joaquim Custódio da Silva Júnior ${ }^{1}$, Rebeca Rizzo ${ }^{1}$
}

\begin{abstract}
RESUMO
Objetivo: analisar a prevalência da gonorréia, infecção por clamídia, sífilis e infecção por HIV entre as mulheres de uma clinica de planejamento familiar em função da presença de sintomas de DST e de comportamentos de risco.

Métodos: mulheres com as idades entre 18 e 30 anos que freqüentavam os serviços de uma clínica de planejamento familiar da rede pública no Brasil foram testadas para a gonorréia e infecção por clamídia, com o uso do teste de amplificação do DNA na urina, para a sífilis e a infecção por HIV por meio de exames de sangue. Foram feitas a todas as participantes perguntas sobre comportamento de demanda de serviços de saúde, a presença de sintomas de DST e comportamentos de risco para as doenças sexualmente transmissiveis.

Resultados: a infecção por clamídia foi encontrada em 11,4\%, a sifilis em 2\%, a gonorréia em $0,5 \%$ e a infecção por HIV em 3\%. Aproximadamente $60 \%$ das mulheres que estavam infectadas por clamidia não apresentavam sintomas. Mulheres que nunca usavam preservativos apresentaram um risco de DST muito mais alto do que aquelas que sempre ou na maioria das vezes usavam preservativos. Houve tendência para as mulheres que nunca haviam feito uso de qualquer método anticoncepcional de apresentar risco mais alto para as DST do que as mulheres que usavam um método anticoncepcional $(p=0,09)$. Muito poucas mulheres reportaram problemas com o uso de álcool ou de drogas ilegais, mas entre aquelas que reportaram tal uso, o risco de DST foi muito alto, particularmente para o uso de maconha. Conclusões: os achados mais significativos foram as altas taxas de doenças numa população de mulheres que reportaram de modo geral comportamentos de baixo risco de saúde. Com base nos nossos achados é essencial que se ofereça o rastreamento de DST/HIV a todas as mulheres com menos de 30 anos que visitam uma clinica de planejamento familiar. Se não se fizer esse rastreamento mais da metade das mulheres infectadas não serão identificadas ou tratadas. Considerando-se a alta sensibilidade e especificidade da nova tecnologia disponivel para o rastreamento da infecção por clamídia, gonorréia e infecção por HIV, e a facilidade de se coletarem espécimes de urina para o diagnóstico, mais esforços devem ser dirigidos para a vigilância das populações de risco, para que a prática clínica corrente possa refletir o risco verdadeiro das populações servidas.
\end{abstract}

PALAVRAS-CHAVE: Rastreamento para DST. Planejamento familiar. Sifilis. Gonorréia. AIDS. Clamidia.

\footnotetext{
${ }^{1}$ Departamento de Ginecologia Obstetrícia e Reprodução Humana, Faculdade de Medicina da UFBa

2 Department of Public Health and Preventive Medicine, Louisiana State University School of Health Sciences

Correspondência:

José S. de Codes

Rua Estácio Gonzaga, 640

40295-020 - Salvador - Bahia

e-mail: josecods@uol.com.br
}

\section{Introdução}

O desenvolvimento de protocolos e normas de assistência padrão para usuários de planejamento familiar depende sobretudo do conhecimento da prevalência de condições médicas específi- 
cas, da tradição de serviços e dos recursos assistenciais disponiveis.

A prestação de serviços de planejamento familiar é um componente importante da assistência à saúde reprodutiva, com o objetivo primordial de retardar ou evitar a gravidez. O enfoque principal de uma consulta típica a um serviço de planejamento familiar é a seleção e discussão de um método anticoncepcional adequado para a mulher que procura o serviço. O método escolhido deverá responder às suas necessidades individuais, desejos e condições fisiológicas, mas dependerá no final dos recursos existentes e do que pode ser gasto por ela ou pelo sistema.

O objetivo secundário da consulta de planejamento familiar é a verificação e assistência a qualquer sintoma incomum concorrente relacionado à saúde reprodutiva. A entrevista típica para a coleta da história médica questiona as usuárias sobre a presença de sintomas anormais relacionados à menstruação, atividade sexual e às doenças sexualmente transmissiveis.

Nos Estados Unidos, uma variedade de estudos realizados têm demonstrado que as infecções por clamidia e gonococo têm alta prevalência entre as usuárias de planejamento familiar, variando de $4,5 \%$ a $12,4 \%$ para a clamídia ${ }^{1,2}$ e $0,5 \%$ para a gonorréia ${ }^{1}$. Devido ao fato de a infecção por clamidia quando não tratada poder apresentar graves conseqüências, como a doença inflamatória pélvica, infertilidade e transmissão neonatal ${ }^{3}$, as normas padrões dos serviços de planejamento familiar nos Estados Unidos foram expandidas para incluir o exame de rotina para essas doenças.

Após a implementação rotineira desses exames por mais de 10 anos, observou-se em algumas regiões dos Estados Unidos um declínio significativo na prevalência de infecção por clamidia e gonorréia ${ }^{4}$. De fato, estudos de custo-efetividade de exames para infecção por clamídia têm demonstrado que para cada dólar desembolsado em um exame existe uma economia de 12 dólares que seriam despendidos no tratamento das conseqüências de uma infecção a longo prazo ${ }^{5,6}$. No Brasil, por outro lado, a detecção das DSTs não é um procedimento de rotina, sendo efetuada apenas quando as mulheres apresentam sintomas, e se há recursos disponiveis para o processamento dos espécimes.

A prevalência elevada de DSTs entre as mulheres nos paises em desenvolvimento tem sugerido a necessidade da expansão dos métodos de detecção ${ }^{7,8}$. Existem presentemente poucos dados sobre a prevalência de DSTs no Brasil, principalmente entre as populações de usuárias de clínicas de planejamento familiar. No entanto, a crescente importância da transmissão heterossexual da HIV/AIDS no Brasil sugere a necessidade de se obterem dados epidemiológicos em uma variedade de populações para se determinarem as estratégias de intervenção mais apropriadas ${ }^{9}$. O presente trabalho apresenta os resultados de um estudo epidemiológico efetuado em usuárias de uma clínica de planejamento familiar mantida pelo sistema público de saúde.

\section{Métodos}

Durante o período de um mês, todas as mulheres com idade entre 18 e 30 anos que procuraram a Clínica de Planejamento Familiar da Maternidade Climério de Oliveira, da Universidade Federal da Bahia, foram convidadas a participar de um estudo de detecção de DST. Ofereceu-se às mesmas a oportunidade de serem testadas para gonorréia e infecção por clamídia por meio de amostra de urina, e para sifilis e HIV por exames de sangue.

Utilizou-se a técnica de amplificação do DNA na urina pela reação em cadeia da ligase (ligase chain reaction - LCR), desenvolvida pelo Laboratório Abbott, para a detecção de gonorréia e infecção por clamídia e por eles denominada de LCX. Os testes de amplificação do DNA na urina foram efetuados no Laboratório de Citogenética da Maternidade Climério de Oliveira. Os exames de sangue para sifilis e HIV foram efetuados no Laboratório Central Gonçalo Moniz da Secretaria de Saúde do Estado da Bahia. O diagnóstico de sífilis foi obtido pelo método da floculação (VDRL), sendo fornecida a referida titulação e confirmado quando necessário pelos métodos de hemaglutinação passiva (HTPA) e EIE (ELISA).

O diagnóstico de HIV foi feito por meio de dois testes Elisa, o primeiro da marca Axsym (Laboratório Abbott), efetuado em bancada, e outro da marca Sanofi-Pasteur, efetuado manualmente. Os casos positivos para quaisquer um deles foram testados por meio da imunofluorescência, e as dúvidas remanescentes foram submetidas ao teste Western blot. Todos os casos positivos foram submetidos a uma segunda coleta de sangue para confirmação do exame. Os casos que permaneceram duvidosos foram submetidos a nova coleta para exame 60 dias mais tarde.

A todas as participantes positivas para infecção por clamídia, gonorréia ou sifilis foi oferecido tratamento gratuito na Maternidade Climério de Oliveira. Os casos positivos para HIV foram contactados pelo Serviço Social da Maternidade e encaminhados para a clinica da Secretaria de Saúde do Estado da Bahia especializada no seu tratamento e acompanhamento. Um total de 202 usuárias da clínica concordaram em participar do estudo. A todas as participantes foi aplicado um questionário contendo perguntas sobre o comportamento de procura de serviços de saúde, a pre- 
sença de sintomas de DST e comportamento de risco para DST.

O questionário foi dividido em seções específicas para averiguar o comportamento de demanda das mulheres aos serviços de saúde, a presença de sintomas de DST e o tipo de tratamento efetuado ou não na ocasião, o tipo de comunidade onde habitavam e o seu grau de solidariedade, coesão e capacidade de resolução dos próprios problemas, e por fim uma série de comportamentos de risco já sabidos de estarem associados a indices mais elevados de DST. Calculou-se o risco de contaminação para qualquer tipo de DST pela comparação com determinados comportamentos já demonstrados estarem associados à sua transmissão. As razões de risco foram calculadas pelo uso de Epi-Info e/ou SPSS.

\section{Resultados}

Aproximadamente $75 \%$ das mulheres elegíveis que foram contactadas concordaram em participar do estudo. As mulheres que se recusaram, o fizeram por duas razões principais: ou elas não quiseram se submeter a uma punção venosa por acharem desagradável, ou por não terem tempo para responder o questionário. A média de idades das que responderam a entrevista foi de 23,3, com uma variação de 18 a 31 . Entre estas mulheres, $90(44,6 \%)$ eram solteiras, 43 (21,3\%) eram casadas e $66(32,7 \%)$ mantinham um relacionamento estável. Apenas 64 (31,7\%) das entrevistadas haviam completado o segundo grau de educação e 53
$(26,2 \%)$ eram desempregadas. Na ocasião da entrevista, 71 de 197 (36\%) reportaram pelo menos um sintoma que poderia ser devido a uma DST, incluindo corrimento $(25,2 \%)$, disúria $(18,3 \%)$, úlcera $(3,5 \%)$ ou outro sintoma $(7,9 \%)$.

Apenas $15(7,4 \%)$ mulheres declararam ter tido mais de um parceiro sexual no período precedente de 1 ano, e menos de $1 \%$ tiveram mais de um parceiro sexual nos últimos 30 dias. Apenas 4 mulheres relatavam o uso de drogas ilegais, e apenas 3 de $201(1,5 \%)$ declararam ter problemas com o uso de bebidas alcoólicas. A maioria das mulheres declararam usar métodos anticoncepcionais, sendo o progesterona injetável o mais freqüentemente empregado (99/202, 49\%), seguido pelo uso do preservativo (29\%), da pílula $(21,8 \%)$ e do DIU $(17,8 \%)$. Nenhuma mulher declarou usar diafragmas ou espermaticidas. Entretanto, apenas uma minoria das mulheres afirmaram usar preservativos todas as vezes que mantinham relações sexuais $(9 \%)$. Cerca de um terço das usuárias de pílulas e DIUs também usavam preservativos e apenas $20 \%$ das usuárias de injetáveis também as usavam.

As mulheres estudadas apresentaram altas taxas de DSTs. Encontrou-se infecção por clamídia em $11,4 \%$, sifilis em $2 \%$, e gonorréia em $0,5 \%$ das mesmas (Tabela 1). Dezesseis por cento do grupo com menos de 21 anos de idade, $8 \%$ do grupo entre 22 e 25 anos e $11 \%$ das mulheres entre 26 e 30 anos apresentavam testes positivos para infecção por clamídia. Dos 4 casos encontrados positivos para sifilis, 3 pertenciam ao grupo entre 22 e 25 anos (4\%) e o quarto pertencia ao grupo entre 26 e 30 anos de idade ( $2 \%$ do grupo).

Tabela 1 - Dados demográficos dos participantes de acordo com DST detectadas

\begin{tabular}{|c|c|c|c|c|}
\hline & Clamídia & Gonorréia & Sífilis & HIV \\
\hline Total $^{*}$ & $23 / 202(11 \%)$ & $1 / 202(0.5 \%)$ & $4 / 202(2 \%)$ & $6 / 202(3 \%)$ \\
\hline \multicolumn{5}{|l|}{ Distribuição por Idade } \\
\hline$<21$ & $11 / 70(16 \%)$ & $0 / 70-$ & $0 / 70-$ & $0 / 70-$ \\
\hline $22-25$ & $6 / 77 \quad(8 \%)$ & $1 / 77 \quad(1 \%)$ & $3 / 77 \quad(4 \%)$ & $3 / 77 \quad(4 \%)$ \\
\hline $26-30$ & $6 / 55(11 \%)$ & $0 / 55-$ & $1 / 55 \quad(2 \%)$ & $3 / 55 \quad(6 \%)$ \\
\hline \multicolumn{5}{|l|}{ Estado Marital } \\
\hline Solteira & $10 / 90(11 \%)$ & $1 / 90 \quad(1 \%)$ & $2 / 90 \quad(1 \%)$ & $1 / 90(1 \%)$ \\
\hline Casada & $5 / 43(12 \%)$ & $0 / 43-$ & $1 / 43 \quad(2 \%)$ & $3 / 43(2 \%)$ \\
\hline União estável & $8 / 66(12 \%)$ & $0 / 66-$ & $1 / 66 \quad(2 \%)$ & $2 / 66(2 \%)$ \\
\hline \multicolumn{5}{|l|}{ Educação } \\
\hline Menor que secundária & $18 / 136(13 \%)$ & $1 / 136(1 \%)$ & $2 / 136(2 \%)$ & $2 / 136(2 \%)$ \\
\hline Secundária completa e superior incompleto & $5 / 65 \quad(8 \%)$ & $0 / 65-$ & $2 / 66(3 \%)$ & $4 / 64(6 \%)$ \\
\hline \multicolumn{5}{|l|}{ Emprego } \\
\hline Trabalhando & $8 / 53 \quad(15 \%)$ & $0 / 53-$ & $1 / 53(2 \%)$ & $1 / 53(2 \%)$ \\
\hline Desempregada & $14 / 142(10 \%)$ & $1 / 142(1 \%)$ & $3 / 142(2 \%)$ & $5 / 142(4 \%)$ \\
\hline
\end{tabular}

${ }^{*}$ A soma dos números pode não totalizar 202 para todas as colunas devido a resultados indeterminados

${ }^{* *}$ As diferenças não são estatisticamente significantes. 
Seis mulheres foram positivas para o HIV. Três delas pertenciam ao grupo entre 22 e 25 anos de idade (4\% do grupo) e as três restantes ao grupo entre 26 e 30 anos (6\% do grupo).

A distribuição dos casos positivos para as diversas DST estudadas não apresentou diferenças significantes entre si, quanto ao estado marital, nivel de educação ou situação empregatícia.

A distribuição por nivel de educação mostrou que $13 \%$ das mulheres que não concluíram nivel secundário foram positivas para a infecção por clamídia, em contraposição a $8 \%$ das que tinham educação secundária completa ou superior incompleto. As proporções de casos positivos para o HIV foram de 2 e $4 \%$, respectivamente, para os grupos em questão.

Quanto ao número de parceiros, $16 \%$ das mulheres que tiveram um ou nenhum parceiro no mês anterior e $17 \%$ das que estavam na mesma situação no ano anterior estavam infectadas pela DST.

A presença de HIV foi confirmada como positiva pelo uso do teste Western blot em 3\% das mulheres. Uma mulher positiva para o HIV estava co-infectada com infecção por clamídia, e o único caso positivo para gonorréia também estava infectado com infecção por clamídia.

Das 23 mulheres que estavam com infecção por clamídia, 14 (60,9\%) não apresentavam sintomas. As mulheres que nunca haviam usado preservativos apresentaram risco muito mais elevado de DSTs do que aquelas que sempre ou na maioria das vezes os usavam. Embora não estatisticamente significante, houve uma tendência para as mulheres que nunca usaram anticoncepcionais de apresentarem risco mais alto para DST do que as mulheres que usavam algum tipo de anticoncepcional (riscos relativos de 2,2 contra 1,0; $\mathrm{p}=0,09)$. Entretanto, quando estudado isoladamente, nenhum método anticoncepcional isolado oferece proteção contra DST. Muito poucas mulheres relataram problemas com o uso de álcool ou de drogas ilegais. Mas entre aquelas que declararam tal uso, o risco de DST foi muito alto, para as que faziam o uso de maconha (Cannabis sativa) (risco relativo de 3,3 contra 1,0 para as que não usavam (Tabela 2 ).

\section{Discussão}

Os achados mais significantes do nosso estudo foram as altas taxas de infecção por clamídia $(11 \%)$, sifilis (2\%) e infecção por HIV (3\%) entre uma população de mulheres que de modo geral referiram comportamentos de baixo risco. Considerando que o risco de DST/HIV entre as mulheres está relacionado à infecção dos seus parceiros sexuais, a autodeclaração de risco das mulheres não constitui indicador útil do seu risco. Tendo em vista estes achados torna-se claro que seria útil oferecer a detecção de DST/HIV a todas as mulheres com menos de 30 anos que procuram os serviços de planejamento familiar da rede pública de saúde. Como ainda não temos dados sobre as mulheres com mais de 30 anos, futuros estudos devem investigar as mulheres de todas a faixas de idade para se determinar o limite de idade apropriado que deverá ser estabelecido para esse rastreamento.

A nossa recomendação para se investigar a presença de DST em mulheres na ausência de sintomas está em contraste direto com a prática corrente no Brasil e em muitos países em desenvolvimento $^{7,10}$. Os protocolos existentes limitam a investigação de DST às mulheres que referem sintomas ou apresentam outros riscos de infecção. Se não se investigarem todas as mulheres, mais da metade das infectadas não serão identificadas ou tratadas.

Dadas as altas taxas de infecção por clamídia e a sífilis, a baixa taxa de mulheres com gonorréia encontrada foi surpreendente. A infecção por clamídia, entretanto, tende a ser mais freqüentemente assintomática do que a gonorréia em homens. É possivel que os homens com gonorréia tendam a procurar o tratamento precoce na doença, reduzindo dessa forma as chances de transmissão.

Causaram também surpresa as altas taxas de positividade sorológica para sífilis e infecção por HIV encontradas entre as mulheres da população estudada. Em Nova York, um dos estados mais altamente afetados pelo HIV dos Estados Unidos, a taxa de infecção por HIV entre as mulheres atendidas pelas clínicas de planejamento familiar da rede pública é de cerca de $0,5 \%{ }^{11}$, menos de um sexto do valor da taxa encontrada em Salvador. Este fato necessita de ser visto com cautela, desde que a pequena amostra escolhida em Salvador determina um amplo intervalo de confiança, de modo que a taxa real da infecção pode ser mais alta ou consideravelmente mais baixa. Essa taxa é ainda menor do que as taxas dos países altamente afetados da África sub-Saariana ${ }^{12}$, mas é necessário que se faça um estudo mais amplo. Além do mais, tendo em vista a alta taxa de infecção por clamídia, devem-se procurar intervenções para reduzir os comportamentos de risco nessa população. 
Tabela 2 - Prevalência de DST e comportamentos de risco.

\begin{tabular}{|c|c|c|c|}
\hline Comportamentos de risco & Número de infectadas $(\%)^{*}$ & Risco relativo & Intervalo de confiança $95 \%$ \\
\hline \multicolumn{4}{|l|}{ Número de parceiros sexuais } \\
\hline 0-1 no mês passado & $32 / 199(16 \%)$ & $N / A$ & \\
\hline$=2$ no mês passado & $0 / 2-$ & & \\
\hline $0-1$ no ano passado & $32 / 186(17 \%)$ & & \\
\hline$=2$ no ano passado & $0 / 15-$ & & \\
\hline \multicolumn{4}{|l|}{ Uso de preservativo } \\
\hline Sempre & $1 / 19 \quad(5 \%)$ & 0,6 & $0,08-4,81$ \\
\hline Algumas vezes & $7 / 85 \quad(8 \%)$ & 1,0 & \\
\hline Nunca & $24 / 96(25 \%)$ & 3,0 & $1,38-6,69$ \\
\hline \multicolumn{4}{|l|}{ Uso na última relação sexual } \\
\hline Sim & $2 / 49 \quad(4 \%)$ & 1,0 & \\
\hline Não & $30 / 152(20 \%)$ & 4,8 & $1,20-19,51$ \\
\hline \multicolumn{4}{|c|}{ Uso de métodos anticoncepcionais } \\
\hline Nenhum método & $4 / 12(33 \%)$ & 2,2 & $0,94-5,37$ \\
\hline Qualquer método & $28 / 189(15 \%)$ & 1,0 & \\
\hline \multicolumn{4}{|l|}{ Pílula } \\
\hline Sim & $4 / 44 \quad(9 \%)$ & 0,5 & $0,19-1,38$ \\
\hline Não & $28 / 157(18 \%)$ & 1,0 & \\
\hline \multicolumn{4}{|l|}{ DIU } \\
\hline Sim & $4 / 36 \quad(11 \%)$ & 0,7 & $0,27-1,99$ \\
\hline Não & $28 / 164(17 \%)$ & 1,0 & \\
\hline \multicolumn{4}{|l|}{ Progesterona injetável } \\
\hline Sim & $17 / 98 \quad(18 \%)$ & 1,2 & $0,63-2,25$ \\
\hline Não & $15 / 103(15 \%)$ & 1,0 & \\
\hline \multicolumn{4}{|l|}{ Preservativo } \\
\hline Sim & $6 / 59 \quad(10 \%)$ & 0,5 & $0,24-1,28$ \\
\hline Não & $26 / 142 \quad(18 \%)$ & 1,0 & \\
\hline \multicolumn{4}{|l|}{ Uso de drogas álcool } \\
\hline Sim & $21 / 132 \quad(16 \%)$ & 1,0 & $0,51-1,95$ \\
\hline Não & $11 / 69 \quad(15 \%)$ & 1,0 & \\
\hline \multicolumn{4}{|c|}{ Problemas c/ o uso de bebidas alcoólicas } \\
\hline $\operatorname{Sim}$ & $(33 \%)$ & 2,1 & $0,42-10,90$ \\
\hline Não & $31 / 198 \quad(16 \%)$ & 1,0 & \\
\hline \multicolumn{4}{|l|}{ Maconha } \\
\hline Sim & $(67 \%)$ & 3,3 & $1,44-7,74$ \\
\hline Não & $28 / 195 \quad(14 \%)$ & 1.0 & \\
\hline
\end{tabular}

${ }^{*}$ A soma das alternativas pode ser menor que 0 total de mulheres por falta de resposta a alguns itens.

Tendo em vista a existência de tecnologia sensivel e específica para a detecção da infecção por clamídia, gonorréia e HIV, e a facilidade de se coletarem espécimes de urina para o diagnóstico das duas primeiras, maiores esforços devem ser dirigidos para a vigilância sanitária das populações de risco, de modo que a prática clínica corrente possa responder ao risco real das populações que estão sendo servidas. Esperamos que os nossos achados sejam aplicados aos protocolos das práticas correntes e que novas determinações do nível de risco da população continuem a ser transmitidas aos serviços médicos, para benefício dos nossos pacientes. 


\section{ABSTRACT}

Purpose: to analyze the prevalence of gonorrhea, Chlamydia, syphilis and HIV among patients attending a family planning clinic regarding presence of STD symptoms and risk behaviors.

Methods: women between the ages of 18 and 30 years who attended a public family planning clinic in Brazil were tested for gonorrhea and Chlamydia using the urine-based DNA amplification test (LCR, Abbott), and a blood test for syphilis (VDRL) and HIV. All participants were asked questions about their health care seeking behavior, the presence of STD symptoms, and about the STD risk behaviors.

Results: Chlamydia was found in $11.4 \%$, syphilis in $2 \%$, gonorrhea in $0.5 \%$ and HIV was confirmed positive in $3 \%$. Approximately $61 \%$ of the women who were infected with Chlamydia had no symptoms. Women who never used condoms had much higher risks for STD than women who used them always or most of the time. Although not statistically significant, there was a trend for women who never used any contraceptive to have a higher risk for STD than women who used some method of contraception $(p=0.09)$. However, when examining separately each contraceptive, none of them alone offered protection against STD. Very few women reported problems related to the use of alcohol or illegal drugs. But among those who did report such use, the risk for STD was very high, particularly regarding marijuana use.

Conclusions: the most significant findings in our study were the high STD rates among a population of women generally reporting low-risk health behaviors. Based upon our findings it is crucial to offer STD/HIV screening to all women under 30 years who visit public family planning clinics. Without screening all women, more than half of the infected women will never be identified or treated. Given the new sensitive and specific technology available to screen for Chlamydia, gonorrhea, and HIV, and the ease of collecting urine specimens for diagnosis, more efforts should be directed to surveillance of populations at risk, so that current clinical practice may reflect the true risk of the populations.

KEY WORDS: STD/HIV screening. Family planning. Chlamydia infection. Syphilis.

\section{Agradecimentos}

Ao Laboratório Abbott, Fulbright Foundation, Laboratório Central Gonçalo Moniz da Secretaria de Saúde do Estado da Bahia, Pathfinder do Brasil e Maternidade Climério de Oliveira da UFBa, a quem expressamos o nosso reconhecimento.

\section{Referências}

1. Gershman KA, Barrow JC. A tale of two sexually transmitted diseases. Prevalences and predictors of chlamydia and gonorrhea in women attending Colorado family planning clinics. Sex Transm Dis 1996; 23:481-8.

2. Addiss DG, Vaughn ML, Golubjatnikov R, Pfister J, Kurtycz DF, Davis JP. Chlamydia trachomatis infection in women attending urban midwestern family planning and community health clinics: risk factors, selective screening, and evaluation of non-culture techniques. Sex Transm Dis 1990; $17: 138-46$.

3. Chlamydia trachomatis genital infections - United States, 1995. MMWR Morb Mortal Wkly Rep 1997; 46:193-8.

4. Mosure DJ, Berman S, Fine D, DeLisle S, Cates W Jr, Boring JR 3rd. Genital chlamydia infections in sexually active female adolescents: do we really need to screen everyone? J Adolesc Health 1997; 20:6-13.

5. The Hidden Epidemic: Confronting Sexually Transmitted Diseases. Eng TR, Butler W, editors. Committee on Prevetion and Control of Sexually Transmitted Diseases, Institute of Medicine; 1997. p. 250.

6. Howell MR, Quinn TC, Brathwaite W, Gaydos CA. Screening women for Chlamydia trachomatis in family planning clinics: the cost-effectiveness of DNA amplification assays. Sex Transm Dis 1998; 25:108-17.

7. Temmerman M, Kidula N, Tyndall M, RukariaKaumbutho R, Muchiri L, Ndinya-Achola JO. The supermarket for women's reproductive health: the burden of genital infections in a family planning clinic in Nairobi, Kenya. Sex Transm Infect 1998; 74:202-4.

8. Wilkinson D, Ndovela N, Harrison A, Lurie M, Connolly C, Sturm AW. Family planning services in developing countries: an opportunity to treat asymptomatic and unrecognised genital tract infections? Genitourin Med 1997; 73:558-60.

9. Giffin K, Lowndes CM. Gender, sexuality, and the prevention of sexually transmissible diseases: a Brazilian study of clinical practice. Soc Sci Med 1999; 48:283-92.

10.Wasti S, Ashfaq MK, Ishaq R, Hamid R. Prevalence of chlamydial infection in females attending antenatal and family planning clinics in Karachi, Pakistan. Aust N Z J Obstet Gynaecol 1997; 37:462-5.

11.Stricof RL, Nattell TC, Novick LF. HIV seroprevalence in clients of sentinel family planning clinics. Am J Public Health 1991; 81 Suppl:41-5.

12.Wilkinson D, Abdool Karim SS, Harrison A, et al. Unrecognized sexually transmitted infections in rural South African women: a hidden epidemic. Bull World Health Org 1999; 77:22-8.

Recebido em: 19/10/01

Aceito com modificações em: 14/03/02 